PACS 33.20.-t

Glushkov A.V., Kovalchuk V.V., Sofronkov A.N., Svinarenko A.A.

Odesa State Environmental University, 15, Lvovskaya str., Odesa, 65016

E-mail: odeku.intsci@gmail.com

\title{
OPTIMIZED QUASIPARTICLE DENSITY FUNCTIONAL APPROACH FOR MULTIELECTRON ATOMIC SYSTEMS
}

\begin{abstract}
We present the optimized version of the quasiparticle density functional theory (DFT), constructed on the principles of the Landau-Migdal Fermi-liquids theory and principles of the optimized one-quasiparticle representation in theory of multielectron systems. The master equations can be naturally obtained on the basis of variational principle, starting from a Lagrangian of an atomic system as a functional of three quasiparticle densities. These densities are similar to the Hartree-Fock (HF) electron density and kinetical energy density correspondingly, however the third density has no an analog in the Hartree-Fock or the standard DFT theory and appears as result of account for the energy dependence of the mass operator $\Sigma$. The elaborated approach to construction of the eigen-functions basis can be characterized as an improved one in comparison with similar basises of other one-particle representations, namely, in the HF, the standard Kohn-Sham approximations etc.
\end{abstract}

1. At present time a density functional theory (DFT) became by a powerful tool in studying the electron structure of different materials, including atomic and molecular systems, solids, semiconductors etc. [1-42]. A construction of the correct energy functionals of a density for multi-body systems represents very actual and important problem of the modern theory of semiconductors and solids, thermodynamics, statistical physics (including a theory of non-equilibrium thermodynamical processes), quantum mechanics and others.

In last time a development of formalism of the energy density functional has been considered in many papers (see [1-7]). Its application is indeed based on the two known theorems by Hohenbreg-Kohn $(\tau=0$, where $\tau$ is a temperature) and Mermin $(\tau \neq 0)[1,2]$. According to these theorems, an energy and thermodynamical potential of the multi-body system are universal density functionals. Though these theorems predict an existence of such a density functional, however its practical realization is connected with a number of the significant difficulties (see [1$3,8-17])$. The problem is complicated under consideration of the non-stationary tasks (the known theorem by Runge-Gross about 1-1 mapping between time-dependent densities and the external potentials [2]).
Let us remind some important results of the density functional theory. It should be mentioned a constructive approach to delivering optimal representations for an exact density functional [1,2,8-16], which has been used for generalization of the HohenbergKohn theorem in order to get an effective density functional for large molecules. As alternative version one could consider a quasiparticle conception of Kohn-Sham and the Levi-Valone method [2,3]. In fact it has been done an attempt practically to realize an idea of the Hohenberg-Kohn theorem.

More advanced analogous approach with account of the multi-particle correlations is developed in ref. $[8,17,18]$.

The quasiparticle Fermi-liquid version of the DFT has been earlier developed in Refs. [1-3,8,17] and based on the principles of the Landau-Migdal Fermi liquids theory. In refs. [4,5] it has been firstly developed a consistent relativistic quasiparticle theory of a density functional formalism and constructed an optimized one-quasiparticle representation in a theory of multi-electron systems. The lowest order multi-body effects, in particular, the gauge dependent radiative contribution for the certain class of the photon propagators calibration are treated in QED formulation and new density functional integral-differential equations are derived. The 
minimal value of the gauge dependent radiative contribution is considered to be the typical representative of the multi-electron correlation effects, whose minimization is a reasonable criteria in the searching for the optimal QED perturbation theory oneelectron basis. In this paper we present the optimized version of the quasiparticle DFT (a Fermi-liquid version of the DFT), based on the principles of the Landau-Migdal Fermiliquids theory and performance of the gauge invariant principle.

The elaborated approach to construction of the eigen-functions basis can be characterized as an improved one in comparison with similar basises of other one-particle representations, namely, in the HF, the standard Kohn-Sham approximations [12-17] etc.

Below we present only the key points of the theory for multielectron atomic systems.

2. According to Refs. [1-5], the master equations can be obtained on the basis of variational principle, if we start from a Lagrangian of an atomic system $L_{q}$. It should be defined as a functional of quasiparticle densities:

$$
\begin{gathered}
v_{0}(r)=\sum_{\lambda} n_{\lambda}\left|\Phi_{\lambda}(r)\right|^{2}, \\
v_{1}(r)=\sum_{\lambda} n_{\lambda}\left|\nabla \Phi_{\lambda}(r)\right|^{2}, \\
v_{2}(r)=\sum_{\lambda} n_{\lambda}\left[\Phi_{\lambda}^{*} \Phi_{\lambda}-\Phi_{\lambda}^{*} \Phi_{\lambda}\right] .
\end{gathered}
$$

The densities $v_{0}$ and $v_{1}$ are similar to the HF electron density and kinetical energy density correspondingly; the density $v_{2}$ has no an analog in the Hartree-Fock (HF) or the standard DFT theory and appears as result of account for the energy dependence of the mass operator $\Sigma$. The functions $\Phi_{\lambda}$ are the solutions of the master equations for multielectron atomic systems with a nuclear charge $\mathrm{Z}$ (in atomic units) as follows:

$$
\begin{aligned}
& {\left[p^{2} / 2-\sum_{\alpha} Z_{\alpha} / r_{\alpha}+\sum_{0}(x)+\right.} \\
& \left.p\left(\partial \sum / \partial p^{2}\right) p\right] \Phi_{\lambda}(x)= \\
& \left(1-\partial \sum / \partial \varepsilon\right) \varepsilon_{\lambda} \Phi_{\lambda}(x)
\end{aligned}
$$

The functions $\Phi_{\lambda}$ in (5) are orthogonal with a weight

$$
\rho_{k}^{-1}=a^{-1}=\left[1-\partial \sum / \partial \varepsilon\right] .
$$

Now one can introduce the wave functions of the quasiparticles

$$
\varphi_{\lambda}=a^{-1 / 2} \Phi_{\lambda}
$$

which are, as usually, orthogonal with weight 1 . For complete definition of $\left\{\varphi_{\lambda}\right\}$ it should be determined the values $\sum_{0}, \partial \sum / \partial p^{2}, \partial \sum / \partial \varepsilon$.

A Lagrangian $L_{q}$ can be written as a sum of a free Lagrangian and Lagrangian of interaction:

$$
L_{q}=L_{q}{ }^{0}+L_{q}{ }^{i n t},
$$

where a free Lagrangian $L_{q}^{0}$ has a standard form:

$$
L_{q}^{0}=\int d r \sum_{\lambda} n_{\lambda} \Phi_{\lambda}^{*}\left(i \partial / \partial t-\varepsilon_{p}\right) \Phi_{\lambda},
$$

The interaction Lagrangian is defined in the form, which is characteristic for a standard (Kohn-Sham) density functional theory (as a sum of the Coulomb and exchangecorrelation terms), however, it takes into account for the energy dependence of a mass operator $\sum$ :

$L_{q}^{\mathrm{int}}=L_{K}-\frac{1}{2} \sum_{i, k=0}^{2} \int_{i k} \beta_{i k} F\left(r_{1}, r_{2}\right) v_{i}\left(r_{1}\right) v_{k}\left(r_{2}\right) d r_{1} d r_{2}$

where $\beta_{i k}$ are some constants (look below), $\mathrm{F}$ is an effective potential of the exchangecorrelation interaction

In the local density approximation in the density functional the potential $F$ can be expressed through the exchange-correlation pseudo-potential $V_{X C}$ as follows [1-5]:

$$
F\left(r_{1,} r_{2}\right)=\delta V_{X C} / \delta v_{0} \cdot \delta\left(r_{1}-r_{2}\right)
$$

According to ref. [1-4], one can get the following expressions for $\sum_{i}=-\delta L_{q}^{\mathrm{int}} / \delta v_{1}$ : 


$$
\begin{aligned}
& \sum_{0}=\left(1-\sum_{e}\right) V_{K}+\sum_{0}^{e x}+ \\
& \frac{1}{2} \beta_{00} \delta^{2} V_{X C} / \delta v^{2} \cdot v_{0}^{2}+\beta_{00} \delta V_{X C} / \delta v_{0} \cdot v_{0}+ \\
& +\beta_{01} \delta V_{X C} / \delta v_{0} \cdot v_{1}+\beta_{01} \delta^{2} V_{X C} / \delta v_{0}^{2} \cdot v_{0} v_{1}+ \\
& \beta_{02} \delta^{2} V_{X C} / \delta v_{0}^{2} \cdot v_{0} v_{2}+\beta_{02} \delta V_{X C} / \delta v_{0} \cdot v_{2} \\
& \sum_{1}=\beta_{01} \delta V_{X C} / \delta v_{0} \cdot v_{0}+ \\
& +\beta_{12} \delta V_{X C} / \delta v_{0} \cdot v_{2}+\beta_{11} \delta V_{X C} / \delta v_{0} \cdot v_{1} ; \\
& \sum_{2}=\beta_{02} \delta V_{X C} / \delta v_{0} \cdot v_{0}+ \\
& +\beta_{12} \delta V_{X C} / \delta v_{0} \cdot v_{1}+\beta_{22} \delta V_{X C} / \delta v_{0} \cdot v_{2} ;
\end{aligned}
$$

Here $V_{K}$ is the Coulomb term (look above), $\sum_{0}^{e x}$ is the exchange term. Using the known canonical relationship:

$$
H_{q}=\Phi_{\lambda}^{*} \delta L_{q} / \delta \Phi_{\lambda}^{*}+\Phi_{\lambda} \delta L_{q} / \delta \Phi_{\lambda}-L_{q}
$$

after some transformations one can receive the expression for the quasiparticle Hamiltonian, which is corresponding to a Lagrangian $L_{q}$ :

$$
\begin{aligned}
& H_{q}=H_{q}^{0}+H_{q}^{\mathrm{int}}=H_{q}^{0}-L_{K}+ \\
& +\frac{1}{2} \beta_{00} \delta V_{X C} / \delta v_{0} \cdot v_{0}^{2}+ \\
& +\beta_{01} \delta V_{X C} / \delta v_{0} \cdot v_{0} \cdot v_{1}+ \\
& \frac{1}{2} \beta_{11} \delta V_{X C} / \delta v_{0} \cdot v_{1}^{2}- \\
& -\frac{1}{2} \beta_{22} \delta V_{X C} / \delta v_{0} \cdot v_{2}^{2}
\end{aligned}
$$

It is obvious that omitting the energy dependence of the mass operator (i.e. supposing $\beta_{02}=0$ ) the quasiparticle density functional theory can be resulted in the standard KohnSham theory.
Further let us give the corresponding comments regarding the constants $\beta_{i k}$. Without a detailed explanation, we note here that the corresponding constants in our theory approximately possess the same universality as ones in the Landau Fermi-liquid theory and Migdal finite Fermi-systems theory. Though it is well known that the entire universality is absent. First of all, it is obvious that the terms with constants $\beta_{01}, \beta_{11}, \beta_{12}, \beta_{22}$ give omitted contribution to the energy functional (at least in the zeroth approximation in comparison with others), so they can be equal to zero in the simple approximation. The value for a constant $\beta_{00}$ in some degree is dependent upon the definition of the potential $V_{X C}$. If as $V_{X C}$ it is use one of the correct exchange-correlation potentials from the standard density functional theory, then without losing a community of statement, the constant $\beta_{00}$ can be equal to 1 . The constant $\beta_{02}$ can be in principle calculated by analytical way, but it is very useful to remember its connection with a spectroscopic factor $F_{s p}$ of atomic or molecular system (it is usually defined from the ionization cross-sections) [5]:

$$
F_{s p}=\left\{1-\frac{\partial}{\partial \in} \sum_{k k}\left[-(I . P .)_{k}\right]\right\}
$$

where I.P. is a ionization potential. It is easily to understand the this definition is in fact corresponding to the pole strength of the corresponding Green's function [62]. The simple approximation for the I.P. is as follows [2-4]:

$$
\begin{gathered}
(I . P .)_{k}=-\left(\epsilon_{k}+F_{k}\right), \\
F_{k}=\Sigma_{k k}\left(-(. I . P .)_{k}\right) \approx \frac{1}{1-\partial \Sigma_{k k}\left(\epsilon_{k}\right) / \partial_{\epsilon}} \Sigma_{k k}\left(\epsilon_{k}\right)
\end{gathered}
$$

It is well known that can be determined by the following standard expression (in the second order of the perturbation theory): 
$\sum_{k k}^{(2)}(\epsilon)=\sum_{\substack{i, j \\ s \in F}} \frac{\left(V_{k s i j}-V_{k s j i}\right) V_{k s i j}}{\epsilon+\epsilon_{s}-\epsilon_{i}-\epsilon_{j}}+\sum_{\substack{i, j \\ s \notin F}} \frac{\left(V_{k s i j}-V_{k s j i}\right) V_{k s i j}}{\epsilon+\epsilon_{s}-\epsilon_{i}-\epsilon_{j}}$

Other details can be found in Refs. [1-10]).

3. As application of the quasiparticle theory we present the estimates for the atomic spectroscopic factors. Using the above written formula, one can simply define the values (3)-(12) one could the quantity (12). In the concrete calculation as potential $V_{X C}$ we use the exchange-correlation pseudo-potential which contains the correlation (GunnarssonLundqvist) potential and relativistic exchanger Kohn-Sham one [4,5].

As example in table 1 we present our calculational data for spectroscopic factors of some atoms.

Table 1.Spectroscopic factors of the external $\mathrm{ns}^{2}$ shells of some atoms and ions

\begin{tabular}{|l|l|l|l|l|l|}
\hline $\begin{array}{l}\text { Atom, } \\
\text { ion }\end{array}$ & $\mathrm{N}$ & $\mathrm{F}_{\mathrm{sp}}$ & $\begin{array}{l}\text { Atom, } \\
\text { ion }\end{array}$ & $\mathrm{n}$ & $\mathrm{F}_{\mathrm{sp}}$ \\
\hline $\mathrm{Ar}$ & 3 & 0.58 & $\mathrm{As}$ & 6 & 0.30 \\
\hline Exp. & 3 & 0.56 & $\mathrm{As}^{-}$ & 6 & 0.31 \\
\hline $\mathrm{RPA}$ & 3 & 0.70 & $\mathrm{Rn}$ & 6 & 0.29 \\
\hline $\mathrm{TI}^{(\mathrm{IV})}$ & 3 & 0.60 & $\mathrm{Fr}^{+}$ & 6 & 0.28 \\
\hline $\mathrm{Xe}$ & 6 & 0.36 & $\mathrm{Fr}$ & 6 & 0.28 \\
\hline $\mathrm{TI}$ & 6 & 0.36 & $\mathrm{Ra}$ & 7 & 0.43 \\
\hline $\mathrm{Pb}$ & 6 & 0.34 & $\mathrm{Ac}$ & 7 & 0.42 \\
\hline $\mathrm{Bi}$ & 6 & 0.33 & $\mathrm{Th}$ & 7 & 0.42 \\
\hline $\mathrm{Po}+$ & 6 & 0.31 & $\mathrm{~Pa}$ & 7 & 0.42 \\
\hline $\mathrm{Po}$ & 6 & 0.31 & $\mathrm{U}$ & 7 & 0.42 \\
\hline
\end{tabular}

There are also listed for the argon atom the experimental value of the spectroscopic factor and the value, obtained in the random phase approximation (RPA) with exchange. It should be noted that the Hartree-Fock theory gives the value of 1 . In conclusion let us remind that the presented approach to definition of the functions basis $\left\{\Phi_{\lambda}\right\}$ of a Hamiltonian $H_{q}$ can be treated as an improved in comparison with similar basises of other oneparticle representations (for example, the HF, the Hatree-Fock-Slater, the standard KohnSham approximations etc.). Naturally, this advancement can be manifested during studying those properties of the multielectron systems, when an accurate account for the complex exchange-correlation effects, including the continuum pressure, energy dependence of a mass operator etc, is critically important [28-40].

\section{References}

1. Glushkov A.V., New approach to theoretical definition of ionization potentials for molecules on the basis of Green's function method. Journ.of Phys.Chem.-1992, 66, 2671-2677.

2. Glushkov A.V., An universal quasiparticle energy functional in a density functional theory for relativistic atom. Optics and Spectr. 1989, 66, 31-36.

3. Glushkov A.V., Quasiparticle approach in the density functional theory under finte temperatures and dynamics of effective Bose -condensate. Ukr. Phys. Journ., 1993, 38(8), 152-157.

4. Glushkov, A.V. Relativistic Quantum theory. Quantum mechanics of atomic systems. Astroprint: Odessa, 2008

5. Glushkov, A.V. Relativistic and correlation effects in spectra of atomic systems. Astroprint: Odessa, 2006.

6. Glushkov A.V., The Green's functions and density functional approach to vibrational structure in the photoelectron spectra of molecules: Review of method// Photoelectronics.-2014, 23, 54-72.

7. Glushkov A.., Koltzova N., Effective account of polarization effects in calculation of oscillator strengths and energies for atoms and molecules by method of equations of motion. Opt. Spectr. 1994, 76(6), 885-890.

8. Khetselius, O.Yu. Quantum structure of electroweak interaction in heavy finite Fermi-systems. Astroprint: Odessa, 2011.

9. Khetselius, O.. Relativistic perturbation theory calculation of the hyperfine structure parameters for some heavy-element isotopes. Int. Journ. Quant.Chem. 2009, 109, 3330-3335.

10. Khetselius, O. Relativistic calculation of the hyperfine structure parameters for 
heavy elements and laser detection of the heavy isotopes. Phys.Scr. 2009, T135, 014023.

11. Khetselius, O.Yu. Hyperfine structure of atomic spectra. Astroprint: Odessa, 2008.

12. Kohn, J.W.; Sham, L.J. Self-Consistent Equations Including Exchange and Correlation Effects. Phys. Rev. A 1964, 140, 1133.

13. Hohenberg, P.; Kohn, W. Inhomogeneous Electron Gas. Phys. Rev. B 1964, 136, 864.

14. Feller, D.; Davidson, E.R. An approximation to frozen natural orbitals through the use of the Hartree-Fock exchange potential. J. Chem. Phys. 1981, 74, 3977-3989.

15. Davidson E.R., Natural Orbitals. $A d v$. Quant. Chem. 1972, 6, 235-266

16. Suaud N.; Malrieu, J.-P. Natural molecular orbitals: limits of a Lowdin's conjecture, Mol. Phys. 2017, 115(21-22), 26842695.

17. Gross E.; Dreizler R. Density Functional Theory; Springer: New York, 1995.

18. Glushkov, A.V. Correction for exchange and correlation effects in multielectron system theory. Journ. of Struct. Chem. 1990, 31(4), 529-532.

19. Köppel H., Domcke W., Cederbaum L.S., Green's function method in quantum chemistry. Adv. Chem. Phys. 1984, 57, 59.

20. Glushkov A.V., Atom in electromagnetic field.-Kiev: KNT, 2005.-450P.

21. Glushkov A.V., Kondratenko P.A., Buyadzhi V., Kvasikova A.S., Shakhman A., Sakun T., Spectroscopy of cooperative laser electron- $\gamma$-nuclear processes in polyatomic molecules. J. of Phys.: Conf. Ser. 2014, 548, 012025.

22. Khetselius, O.Yu. Relativistic Energy Approach to Cooperative Electron- $\gamma$ Nuclear Processes: NEET Effect In Quantum Systems in Chemistry and Physics, Series: Progress in Theoretical Chemistry and Physics; Nishikawa, K., Maruani, J., Brändas, E., Delgado-Barrio, G., Piecuch, P., Eds.; Springer: Dordrecht, 2012; Vol. 26, pp 217-229.

23. Khetselius, O.Yu. Relativistic perturbation theory calculation of the hyperfine structure parameters for some heavyelement isotopes. Int. Journ.Quant.Chem. 2009, 109, 3330-3335.

24. Khetselius, O.Yu. Relativistic calculation of the hyperfine structure parameters for heavy elements and laser detection of the heavy isotopes. Phys.Scripta. 2009, 135, 014023.

25. Khetselius, O.Yu. Optimized Perturbation Theory for Calculating the Hyperfine Line Shift and Broadening of Heavy Atoms in a Buffer Gas. In Frontiers in Quantum Methods and Applications in Chemistry and Physics, Series: Progress in Theoretical Chemistry and Physics; Nascimento, M., Maruani, J., Brändas, E., Delgado-Barrio, G., Eds.; Springer: Cham, 2015; Vol. 29, pp. 55-76.

26. Khetselius, O.Yu. Relativistic calculating the spectral lines hyperfine structure parameters for heavy ions.AIP Conf. Proc. 2008, 1058, 363-365.

27. Khetselius, O.Yu. Hyperfine structure of energy levels for isotopes ${ }^{73} \mathrm{Ge},{ }^{75} \mathrm{As}$, ${ }^{201} \mathrm{Hg}$. Photoelectr. 2007, 16, 129-132

28. Ivanov, L.N.; Ivanova, E.P. Method of Sturm orbitals in calculation of physical characteristics of radiation from atoms and ions. JETP. 1996, 83, 258-266.

29. Glushkov, A.V., Ivanov, L.N. Radiation decay of atomic states: atomic residue polarization and gauge noninvariant contributions. Phys. Lett. A 1992, 170, 33.

30. Glushkov, A.V.; Ivanov, L.N. DC strongfield Stark effect: consistent quantummechanical approach. J. Phys. B: At. Mol. Opt. Phys. 1993, 26, L379-386.

31. Ivanova, E.P., Ivanov, L.N., Glushkov, A., Kramida, A. High order corrections in the relativistic perturbation theory with the model zeroth approximation, $\mathrm{Mg}$ Like and Ne-Like Ions. Phys. Scripta 1985, 32, 513-522.

32. Glushkov, A.V. Multiphoton spectroscopy of atoms and nuclei in a laser field: Relativistic energy approach and radiation atomic lines moments method. Adv. in Quantum Chem. 2019, 78, 253-285.

33. Khetselius, O.Yu. Optimized relativistic 
many-body perturbation theory calculation of wavelengths and oscillator strengths for Li-like multicharged ions. Adv. Quant. Chem. 2019, 78, 223-251.

34. Svinarenko, A., Glushkov, A, Khetselius, O., Ternovsky, V., Dubrovskaya Y., Kuznetsova A., Buyadzhi V. Theoretical spectroscopy of rare-earth elements: spectra and autoionization resonances. Rare Earth Element, Ed. J. Orjuela (InTech). 2017, pp 83-104.

35. Glushkov, A., Khetselius, O., Svinarenko A., Buyadzhi, V., Ternovsky, V. Kuznetsova, A., Bashkarev, P. Relativistic perturbation theory formalism to computing spectra and radiation characteristics: application to heavy element. Recent Studies in Perturbation Theory, ed. D. Uzunov (InTech). 2017, 131-150.

36. Glushkov, A. Spectroscopy of cooperative muon-gamma-nuclear processes: Energy and spectral parameters J. Phys.: Conf. Ser. 2012, 397, 012011.

37. Glushkov, A.V. Spectroscopy of atom and nucleus in a strong laser field: Stark effect and multiphoton resonances. J. Phys.: Conf. Ser. 2014, 548, 012020.
38. Glushkov, A.V., Khetselius, O.Yu., Svinarenko A.A. Theoretical spectroscopy of autoionization resonances in spectra of lanthanides atoms. Phys. Scripta. 2013, T153, 014029.

39. Glushkov, A.V., Svinarenko, A.A., Ternovsky, V.B., Smirnov, A.V., Zaichko, P.A. Spectroscopy of the complex autoionization resonances in spectrum of helium: Test and new spectral data. Photoelectronics. 2015, 24, 94-102.

40. Glushkov, A.V., Ternovsky, V.B., Buyadzhi, V., Zaichko, P., Nikola, L. Advanced relativistic energy approach to radiation decay processes in atomic systems. Photoelectr. 2015, 24, 11-22.

41. Glushkov A., Khetselius O., Kruglyak Yu., Ternovsky V. Calculational Methods in Quantum Geometry and Chaos theory. P.3. Odessa, 2014.

42. Glushkov A., Khetselius O., Svinarenko A, Buyadzhi V. Methods of computational mathematics and mathematical phys. P.1.TES, Odessa, 2015.

PACS 33.20.-t

Glushkov A.V., Kovalchuk V.V., Sofronkov A.N., Svinarenko A.A.

\section{OPTIMIZED QUASIPARTICLE DENSITY FUNCTIONAL APPROACH FOR MULTIELECTRON ATOMIC SYSTEMS}

Summary. We present the optimized version of the quasiparticle density functional theory (DFT), constructed on the principles of the Landau-Migdal Fermi-liquids theory and principles of the optimized one-quasiparticle representation in theory of multielectron systems. The master equations can be naturally obtained on the basis of variational principle, starting from a Lagrangian of an atomic system as a functional of three quasiparticle densities. These densities are similar to the Hartree-Fock (HF) electron density and kinetical energy density correspondingly, however the third density has no an analog in the HartreeFock or the standard DFT theory and appears as result of account for the energy dependence of the mass operator $\Sigma$. The elaborated approach to construction of the eigen-functions basis can be characterized as an improved one in comparison with similar basises of other oneparticle representations, namely, in the HF, the standard Kohn-Sham approximations etc.

Key words: quasiparticle density functional theory, exchange-correlation corrections 
Глушков А.В., Ковальчук В.В., Софронков А.Н., Свинаренко А.А.

\section{ОПТИМИЗИРОВАННАЯ КВАЗИЧАСТИЧНАЯ ТЕОРИЯ ФУНКЦИОНАЛА ПЛОТНОСТИ ДЛЯ МНОГОЭЛЕКТРОННЫХ АТОМНЫХ СИСТЕМ}

Резюме. Представлена оптимизированная версия квазичастичной теории функционала плотности (ТФП), построенная на принципах теории ферми-жидкости ЛандауМигдала и введении оптимального одноквазичастичного представления в теории многоэлектронных систем. Основные уравнения могут быть естественно получены на основе вариационного принципа, исходя из лагранжиана атомной системы, представленного в виде функционала трех квазичастичных плотностей. Последние аналогичны стандартным электронной плотности Хартри-Фока (ХФ) и плотности кинетической энергии; однако третья плотность не имеет аналога в теории ХФ или стандартной ТФП и появляется как результат учета энергетической зависимости массового оператора квазичастиц. Разработанный подход к построению базиса собственных функций представляется более эффективным в сравнении с аналогичными базисами других одночастичных представлений, в частности, в приближениях ХФ или стандартном приближении Кона-Шэма и др.

Ключевые слова: квазичастичная теория функционала плотности, обменнокорреляционные поправки

PACS 33.20.-t

Глушков О.В., Ковальчук В.В., Софронков О.Н., Свинаренко А.А.

\section{ОПТИМІЗОВАНА КВАЗІЧАСТИНКОВА ТЕОРІЯ ФУНКЦІОНАЛА ГУСТИНИ ДЛЯ БАГАТОЕЛЕКТРОННИХ АТОМНИХ СИСТЕМ}

Резюме. Представлена оптимізована версія квазічастинкової теорії функціонала густини (ТФГ), побудованої на принципах теорії фермі-рідини Ландау-Мігдала і введенні оптимального одноквазічастинкового уявлення в теорії багатоелектронних систем. Основні рівняння теорії можуть бути природно отримані на основі варіаційного принципу, виходячи з лагранжіану атомної системи, представленого у вигляді функціоналу трьох квазічастинкових густин. Останні аналогічні стандартним електронній густині Хартрі-Фока (ХФ) і густині кінетичної енергії; однак, третя густина не має аналога в теорії ХФ або стандартній ТФГ і з'являється як результат урахування енергетичної залежності масового оператора квазічастинок. Розроблений підхід до побудови базису власних функцій видається більш ефективним у порівнянні з аналогічними базисами інших одночасткових уявлень, зокрема, в наближеннях ХФ або стандартному наближенні Кона-Шема і ін.

Ключові слова: квазічастинкова теорія функціоналу густини, обміннокореляційні поправки 